\title{
Acoustémologie et empreinte sonore. Faire avec et faire par les sons. Être avec et être par les sons
}

\section{Marie Baltazar et Laurent Legrain}

\section{(2) OpenEdition}

1 Journals

Édition électronique

URL : https://journals.openedition.org/clo/8595

DOI : $10.4000 /$ clo.8595

ISSN : 2266-1816

Éditeur

INALCO

\section{Édition imprimée}

Date de publication : 30 juin 2020

Pagination : 175-194

ISBN : 978-2-85831-392-1

ISSN : 0396-891X

\section{Référence électronique}

Marie Baltazar et Laurent Legrain, «Acoustémologie et empreinte sonore. Faire avec et faire par les sons. Être avec et être par les sons », Cahiers de littérature orale [En ligne], 87 | 2020, mis en ligne le 16 septembre 2021, consulté le 09 novembre 2022. URL : http://journals.openedition.org/clo/8595; DOI : https://doi.org/10.4000/clo.8595

\section{(c) (†) \$}

Creative Commons - Attribution - Pas d'Utilisation Commerciale 4.0 International - CC BY-NC 4.0

https://creativecommons.org/licenses/by-nc/4.0/ 


\section{Acoustémologie et empreinte sonore. Faire avec et faire par les sons. Être avec et être par les sons ${ }^{1}$}

Marie BALTAZAR

CAS-LISST

Laurent LEGRAIN

Université Toulouse Jean Jaurès, CAS-LISST

"[...] just as in a genial conversation, there is a continuous interchange and blending, and yet each speaker not only retains his own character but manifests it more clearly than his wont."

(Dewey, 1934, p. 36-37)

Le pasteur Gilbert White (1720-1793) a écrit des pages magnifiques sur les oiseaux qui nichaient ou qui traversaient sa paroisse de Selborne au sud de l'Angleterre. Le 13 septembre 1774, il confie à l'un des destinataires de ses lettres Daines Barrington, juge à Chester et ornithologue patenté - qu'il souffre de

1. L'ethnomusicologue Steven Feld a plusieurs fois réussi l'impossible comme faire se conjuguer l'analyse structuraliste d'un mythe et la description dense préconisée par Clifford Geertz ou encore écrire l'un des plus beaux livres d'ethnomusicologie et le dédicacer à la mémoire de Charlie Parker - surnommé Bird -, John Coltrane et Charles Mingus - que l'on serait bien en mal de surnommer Bird en les écoutant. Le commun des mortels referme ce genre de livres en se disant « donc c'est possible », et cette simple réflexion le tient un bon moment, le poussant à tenter ou mieux à expérimenter, ou mieux encore à mettre le monde à l'épreuve ; à l'éprouver. 
surdité temporaire et que les « messages agréables et petites suggestions éveillées par les sons de la campagne » (White, 2011, p. 236) lui sont alors inaccessibles. Il s'attriste. Le mois de mai devient aussi muet que le mois d'août. Heureusement, sa vue est bonne mais il perd l'ouïe. Il conclut sa lettre par ce constat : « Et la sagesse se voit refuser une voie d'accès » (ibid.). Les sons ou mieux, les petites suggestions sonores seraient donc, pour notre pasteur, une des voies d'accès à la sagesse ? White qui écrit à la fin du XVIII ${ }^{\mathrm{e}}$ siècle adopte, nous semble-t-il, une ligne de conduite similaire à celle proposée par l'ethnomusicologue Steven Feld pour qui le son est une «modalité de connaissance et d'être au monde » (2003, p. 226), modalité qu'il étiquette d'un néologisme, l'acoustémologie, formé à partir des termes « acoustique » et « épistémologie ». Nous disons « adopte une ligne de conduite » et non pas, par exemple, « adopte une perspective » justement parce que les sons sont des suggestions et comme toutes les suggestions, on peut les prendre en compte ou les laisser s'évanouir. Or, se rendre sensible à une suggestion demande tout un travail, ou plus précisément, nécessite d'adopter une façon de se comporter, de manœuvrer, bref de se conduire. La métaphore de la connaissance comme perspective convie l'image d'une personne gagnant un promontoire puis observant sans bouger ce qu'il veut connaître tandis que celle de la ligne de conduite met l'accent sur toutes les opérations entreprises, autrement dit, tout ce qu'il faut faire pour connaître et être au monde ou mieux «se faire être au monde », même si l'expression est trop lourde et que nous ne la conserverons pas. Les observations de White nous permettront de mettre au travail deux concepts que nous pressentons comme étant intimement liés : l'attachement (Hennion, 2007 ; Latour, 2012) et l'épistémologie acoustique. Ensuite nous y amalgamerons - au sens chimique - celui d'empreinte sonore. Ce travail de mise en relation conceptuelle n'est pas uniquement un jeu de l'esprit. L'enjeu est de ramener dans le champ de la description ethnographique toute une série de procédures et d'opérations souvent minuscules ou « quasi-microbiennes », comme disait Michel de Certeau (1990, XL), par lesquelles les gens se font des mondes et les habitent. Si ce travail de fabrication mobilise des sons, alors il nous intéresse et nous allons chercher à le décrire au mieux à partir de nos terrains respectifs. Depuis l'Angleterre du pasteur White, nous gagnerons la Mongolie en faisant halte en France, dans le Jura, prêtant successivement l'oreille à un écho, aux brames des cerfs, aux crissements de plantes engoncées dans le gel printanier, afin de saisir quelque chose des empreintes laissées par ces sons sur le devenir des personnes rencontrées au fil de nos descriptions. 


\section{Des cris dans la vallée}

Tout commence par ce que nous avons appelé plus haut une « suggestion sonore $\gg$. Elle ne vient d'abord aux oreilles de White qu'indirectement, mais l'anecdote transporte le pasteur au sens figuré comme au sens propre puisque quelque temps plus tard, ayant recouvré l'ouïe, le voilà enthousiaste, testant lui-même l'écho dont lui a parlé un jeune homme de la région. La relation de cette exploration sonore fait l'objet de sa lettre du 12 février 1778 à Daines Barrington :

Dans une région aussi variée que la nôtre, si pleine de belles vallées profondes et de pentes boisées, il n'est pas étonnant qu'il y ait beaucoup d'échos. Nous en avons découvert plusieurs qui renvoient très agréablement les cris d'une meute de chiens, les notes d'un cor de chasse, une sonnerie harmonieuse de cloches ou le chant des oiseaux. Nous n'avions pas encore trouvé d'écho qui reproduise plusieurs syllabes distinctement, jusqu'au jour où un jeune homme qui, par un soir d'été, s'étant éloigné de ses compagnons de promenade, les appelait pour les retrouver, en découvrit par hasard un très curieux dans un endroit fort inattendu. Tout d'abord, il fut très surpris et était convaincu que quelqu'un se moquait de lui. Mais après avoir répété sa tentative en plusieurs langues et s'être rendu compte que son interlocuteur était polyglotte, il finit par percevoir l'illusion.

En une soirée, avant que ne cessent les bruits de la campagne, cet écho pouvait répéter dix syllabes de façon très articulée et distincte, surtout si l'on choisissait des dactyles rapides. Les dernières syllabes de Tityre, tu patule recubans... [Tytire allongé sous les grandes frondaisons...] revenaient au jeune homme aussi audibles et intelligibles que lorsqu'il les déclamait. Sans aucun doute, si l'on avait tenté l'expérience à minuit, lorsque l'air est très élastique et que tout est silencieux, on aurait certainement pu obtenir une ou deux syllabes de plus. À cause de la distance, il était mal commode de faire l'essai aussi tard.

Nous avons remarqué que les dactyles rapides donnaient les meilleurs résultats. En effet, quand nous avons mis les capacités de cet écho à l'épreuve à l'aide de spondées du même nombre de syllabes mais lents, lourds et poussifs tels que Monstrum horrendum, informe ingens... [Monstre horrible informe et immense...] nous n'avons pu en capter que quatre ou cinq. (White, 2011, p. 271-272) 
Dans la suite de sa lettre, White se lance dans une réflexion sur les surfaces les mieux à même de renvoyer l'écho. Dans le feuillage des arbres, souligne-t-il à l'attention de son correspondant, la voix «s'empêtre » et «s'embarrasse », tandis que les rocs dénudés permettent un rebond du son beaucoup plus souple. Il cherche alors la source de l'écho de cette vallée qu'il trouve être un séchoir à houblon taillé à même la pierre un peu en contrebas. Il se lance dans des calculs compliqués visant à localiser le centrum phonicum, soit l'endroit d'où l'on peut tirer le maximum des capacités de résonnance de la vallée. Il procède par essais et erreurs, par déplacements successifs. Ce centre phonique une fois circonscrit - il s'agit du « bord de l'ados abrupt qui domine le creux du chemin charretier » (White, 2011, p. 272-273) - il tente d'établir une loi qui corrèle la puissance d'un écho à la distance qui sépare la source de son centre phonique. Fort de sa démonstration, il entre en contact épistolaire avec le docteur Plot qui s'est intéressé à ce sujet et trouve en lui un « savant de bonne composition » (ibid.), capable d'entendre que la loi qu'il avait personnellement échafaudée et mise en forme dans son Histoire de l'Oxfordshire peut être améliorée par l'introduction d'autres paramètres, comme la nature des surfaces sur lesquelles les sons rebondissent ainsi que l'élasticité de l'air, lui-même fonction de l'heure de la journée et de la température ambiante. White en profite pour s'en prendre aux écrits de Virgile - dont les dactyles lui avaient servi à éprouver l'écho - car le poète avait erronément signalé que les ruchers devaient être situés en dehors des zones à écho. Virgile pensait que les abeilles étaient affectées par ces sons jugés nocifs. Le pasteur de Selborne invoque et s'allie aux « savants de son époque » qui certifient quant à eux que les abeilles ne possèdent pas d'organe auditif. « J'ai mis souvent [mes abeilles] à l'épreuve à l'aide d'un gros porte-voix tenu tout près de leurs ruches, hurlant si fort qu'un navire à un mille de distance aurait pu m'entendre : ces insectes poursuivaient tranquillement leurs diverses activités, sans montrer la moindre sensibilité ni le moindre malaise »(White, 2011, p. 274). On imagine la scène avec délice. Enfin, White mentionne que l'écho a disparu peu après sa découverte du fait de la mise en culture de houblon sur les terres qui s'étendent entre le centre phonique et la source. Le son perd de sa puissance en passant entre les perches qui maintiennent les plants de houblon et il est encore affaibli un peu plus loin par une haie plantée là pour protéger la culture des vents dominants. Notre pasteur tire de tout cela quelques conseils d'aménagement qu'un « gentilhomme fortuné » et sensible d'oreille pourrait mettre à profit pour fabriquer de toute pièce un écho plaisant sur ses terres. 


\section{Attachement et acoustémologie}

De nombreuses dimensions constitutives des notions d'attachement et d'acoustémologie sont lisibles dans la description des efforts de cet homme d'un autre siècle pour appréhender le monde qui entoure sa paroisse et s'y engager. Dans le sillage du travail d'Antoine Hennion et de Bruno Latour, nous envisageons l'attachement comme un processus qui produit dans le même mouvement des personnes attentives aux singularités des choses qui les entourent et un monde chargé de ces singularités agissantes. La notion a pour visée de décrire au mieux le processus qui façonne des personnes attentives et un monde intéressant. Pour le dire simplement à partir de notre exemple, il ne s'agit plus d'une vallée quelque part en Angleterre, il s'agit de cette vallée précise que traverse le chemin qui va de Selborne à Nore-Hill et qui résonne de manière si particulière après que, dans des circonstances elles aussi très particulières, on s'est intéressé à elle et qu'on a mis à l'épreuve ses performances acoustiques. Antoine Hennion (2003) souligne que le développement d'un attachement, quel qu'il soit, convoque toujours les mêmes éléments. On les voit poindre sous la plume de White. Il est utile de les faire apparaître plus précisément pour nous permettre de monter en généralité. Quels sont donc ces éléments?

- Tout d'abord, un jeune homme perdu dans une vallée, qui crie et qui parle à son pasteur des sons qui lui reviennent. Ce même pasteur qui gagne le lieu pour crier et se met en quête du centre phonique de l'écho. Gibert White, toujours lui, qui lit les écrits du docteur Plot, discute des théories de ce dernier et fait un compte rendu de tout cela à son correspondant Daines Barrington. Le processus d'attachement suppose des « relations sociales », soit des relations entre des gens médiées par une attention à un même objet. On ne s'attache jamais seul aux choses. Le processus tire une partie de sa vitalité de sa capacité à générer du lien.

- Puis, une réponse sonore de la vallée aux cris du jeune homme ainsi qu'à ceux de Gilbert White. Une réponse qui révèle une acoustique différente de celles des autres vallées. On pourrait parler de propriétés acoustiques propres à cette vallée tout en retenant bien que ces propriétés sont elles-mêmes le produit d'une mise à l'épreuve et qu'elles sont très dépendantes d'un agencement spatial provisoire, lié à des activités humaines (le séchoir à houblon aménagé par la communauté locale dont on comprend qu'elle n'est probablement pas indifférente au goût et aux effets de la bière). Il y a donc pas mal d'humain dans les propriétés de l'objet comme il y avait pas mal de propriétés de l'écho dans les relations 
humaines qui transpirent de la lettre de White. La fonction phatique de son courrier ne doit pas cacher le fait que ce qui lie ces deux férus d'observation naturaliste, c'est l'exploration de leur campagne respective.

Outre des relations sociales et des objets aux réponses singulières, que faut-il d'autre pour alimenter le processus d'attachement ?

. Un déploiement d'énergie assez impressionnant de la part des personnes impliquées. Surtout Gilbert White qui reste au final celui qui est le plus attaché à cet écho dont il note d'ailleurs la disparition quelque temps plus tard, non sans en tirer des déductions sur les possibilités de canaliser ces «phénomènes acoustiques » pour le plus grand plaisir des gentilshommes fortunés de sa paroisse. Imaginons White crier, se déplacer, crier à nouveau, inscrire quelques notes sur du papier, éventuellement prendre des informations auprès des cultivateurs de houblon, revenir à un autre moment de la journée, crier à nouveau (tout cela sans souci du qu'en dira-t-on...), compléter ses notes, s'installer à son bureau et écrire à son correspondant, etc. Lorsque nous arriverons à nos vignettes ethnographiques nous n'aurons plus besoin d'imaginer tout ce travail. Il sera devant nous. Il nous faudra décrire toutes ces minuscules opérations, ce qui n'est pas spécialement plus simple.

. De l'attachement, il manque une composante absolument indispensable pour boucler le processus. Pour qu'une chose devienne intéressante, il faut en effet que sa mise à l'épreuve provoque des variations de ce que nous ressentons - même si elles sont infimes. Ces «altérations de soi » se lisent en filigrane dans la lettre de White à Barrington : sa surprise, sa joie, son enthousiasme, peut-être aussi sa déception au moment où, de l'ados abrupt qui domine le creux du chemin charretier qui s'en va vers Nore-Hill, il crie sans obtenir de réponse de l'écho. La correspondance de White est truffée de ces altérations dont nous listons encore, ici, quelques affleurements : admiration, stupeur, étonnement, sentiment de respect ou d'étrangeté, amusement, impatience, joie liée à une découverte ou joie (certainement un peu différente) de l'administration d'une preuve, plaisir de l'imagination, gêne, désagrément, contrariété, etc. ${ }^{2}$ La liste pourrait

2. Toutes ces altérations ne peuvent sans doute pas être versées dans cet énorme fourre-tout que nous appelons «émotion », mais on peut suivre John Dewey (1934) lorsqu'il dit que ces mots fixent des processus dont la durée, la croissance et l'atténuation sont des traits définitoires importants. Ces mots qui qualifient des tonalités émotionnelles donnent une qualité unitaire à ce qui est de l'ordre d'un drame qui se développe. 
Acoustémologie et empreinte sonore. Faire aVec et faire par les sons.

ÊTRE AVEC ET ÊTRE PAR LES SONS

Marie BALtazar \& Laurent Legrain

s'allonger mais l'objectif n'est pas de faire gonfler l'intériorité de notre pasteur. Lui-même s'y refuse d'ailleurs. Par bonheur, son journal est plus extime qu'intime et White préfère de loin l'exploration à l'imploration ${ }^{3}$. En listant ce qu'il laisse entrevoir au fil des pages des altérations de sa personne, il s'agit plutôt de montrer que cette mise à l'épreuve de sa chère campagne de Selborne, cette connaissance en construction, implique aussi, chez lui, une transformation, un devenir autre ou, pour le dire en s'aidant d'une métaphore empruntée à l'écrivaine Camille Laurens, une co-naissance (Laurens, 2011), c'est-à-dire une double naissance. Nous voulons parler de l'advenue d'un monde plus riche de singularités d'un côté et d'un individu altéré ou affecté, donc transformé, de l'autre.

Si White avait été un Kaluli vivant sur les contreforts du mont Bosavi en Papouasie-Nouvelle-Guignée, il aurait appelé l'écho gugu-go:go:, terme que Steven Feld verse dans la catégorie des « phonostèmes mimétiques » (Feld, 2003, p. 228) dont la fonction est de rendre compte d'une impression laissée sur le locuteur par une chose ou une situation. En l'occurrence, il s'agit de l'impression laissée par une gamme de sons caractéristiques du paysage sonore de la forêt tropicale qui couvre les piémonts encaissés du mont Bosavi. L'analyse de ce genre de termes est une dimension fondamentale de la notion d'acoustémologie proposée par Feld. L'acoustémologie est née d'un questionnement et d'une volonté d'approfondir les modalités des relations qui existent entre le sens musical d'une population et le paysage sonore au sein duquel elle évolue. La notion voit le jour en réaction à une tendance de l'écologie acoustique à séparer l'étude d'un milieu sonore de son appréhension subjective par ceux qui y baignent. C'est une tendance classique : on circonscrit les deux polarités habituelles de l'objectif (le paysage sonore) et du subjectif (les gens qui le perçoivent) et on cherche ensuite les modalités relationnelles qui les unissent. Feld voudrait repartir de la relation et en cela il partage la prémisse générale à partir de laquelle s'éploie l'étude des attachements. Son point de départ est l'empreinte laissée par les sons de l'environnement sur l'humain qui les entend, les incorpore, et en fait usage dans sa façon de « musiquer », c'est-à-dire sa façon de prendre part à une performance musicale soit en chantant ou en jouant, soit en écoutant (Small, 2019). Ainsi, «gu » évoque un son descendant. En dupliquant le terme, la langue Kaluli dénote le caractère continu d'un processus en cours : « gugu » est donc un son qui

3. L'imploration correspond pour l'écrivain Michel Tournier à un repliement pleurnichard sur de « petits tas de misérables secrets » (Tournier, 2004, p. 12), expression qu'il emprunte à André Malraux. 
descend continuellement. «Go: » évoque un son qui part d'une source et jaillit vers l'extérieur ou vers les hauteurs (profondeur et hauteur sont deux dimensions de la perception que le milieu de la forêt tropicale rend confuses). À nouveau, la duplication du terme convoque l'idée d'un processus continu. L'analyse du vocable permet à Feld de prendre conscience de ce qu'un Kaluli entend dans l'écho, phénomène sonore prédominant dans son environnement : un son qui jaillit (vers l'extérieur et vers les hauteurs) en descendant. Aller vers les hauteurs en descendant est certes paradoxal, mais cette contradiction évoque l'impression de confusion sensorielle que provoque l'écho. Cette manière de concevoir le son est englobée dans un concept plus large, lift-up-over-sounding ${ }^{4}$, qui met l'accent sur la superposition des sons et leurs constantes altérations. Comme l'harmonie sous nos latitudes, lift-up-over-sounding est à la fois un des concepts essentiels de la théorie musicale développée par les Kaluli et un des nombreux fondements du pouvoir expressif et émotionnel prêté au chant.

Il nous semble que la notion d'acoustémologie recèle de potentialités d'exploration plus larges que l'analyse des effets d'un milieu sonore sur les façons de musiquer d'une population. L'acoustémologie est, soulignait Feld, un mode de connaissance et d'être au monde. Le programme que nous aimerions suivre dans la deuxième partie de cet article est tout entier contenu dans les implications d'une proposition corolaire de Steven Feld : Soundscapes are perceived and interpreted by human actors who attend to them as a way of making their place in and through the world (Feld, 2003, p. 226). To attend to ${ }^{5}$ le verbe exprime à la fois la présence, le fait de porter son attention à, de participer, voire de prendre soin, de s'occuper de et de veiller à... soit d'être présent et activement impliqué dans la production de ce à quoi l'on est présent, en l'occurrence ici, le paysage sonore $^{6}$. C'est le jalon qui guidera nos descriptions et ni le paysage sonore, ni

4. Dans ce numéro, lift-up-over-sounding est traduit par «son qui s'élève par-dessus ». Cette traduction nous semble pertinente et nous ne saurions proposer mieux sauf peut-être, de ne pas traduire l'expression et d'ainsi laisser chacun en creuser la complexité en s'y essayant.

5. Yves Citton rappelle qu'étymologiquement notre mot attention comme le verbe anglais to attend to vient du latin ad-tendere qui signifie «tendre vers » (Citton, 2014, p. 232-233).

6. Il nous semble que notre position rejoint celle de l'anthropologue Tim Ingold lorsqu'il définit la notion de l'habiter comme la participation à la formation des choses qui nous entourent, la participation à un monde de forces, de flux et d'énergies (Ingold, 2018, p. 192). Une autre façon de définir l'acoustémologie serait donc de dire qu'elle est la manière dont nous habitons le monde par les sons. 
la musique per se ne seront le centre de notre visée analytique. Les questions qui cadrent nos ethnographies sont les suivantes : comment faire du son un appui ou un soutien dans sa manière d' « être au monde », ou encore comment faire $\mathrm{du}$ son le principe de cheminement d'une connaissance toujours en transit mais aussi toujours plus intime des choses qui nous entourent ? Nous sommes au cœur des processus d'attachement et, dans les deux descriptions qui suivent, nos interlocuteurs vont se charger de nous le rappeler lorsque nous allons, nous les ethnographes censément empathiques, reconstruire le son sans prendre en considération leur propre épistémologie acoustique. Ce sont les impairs que nous avons commis qui vont nous conduire à la notion d'empreinte sonore que nous glisserons dans le sillage des réflexions sur l'attachement et l'acoustémologie déjà posées plus haut. C'est vers le massif du Massacre que la piste nous mène d'abord en suivant l'ethnographie de Marie.

\section{Empreintes sonores}

\section{Bramer}

Septembre 2018, j'ai rendez-vous avec le garde-chasse, un forestier et les chasseurs de la commune de Lajoux, dans le Jura, pour l'opération annuelle de comptage des cerfs sur le massif du Massacre. Pendant deux heures, à la tombée de la nuit, nous allons les écouter bramer : pousser le râle puissant du rut, rauque et profond qui, pendant un mois, de la mi-septembre à la mi-octobre, retentit avec vigueur dans les forêts. Ce soir, chacun prendra un poste d'écoute et notera l'heure et la localisation de chaque brame. Puis nous nous retrouverons pour recouper chaque fiche d'observation afin de positionner les cerfs recensés sur une carte, une opération permettant d'évaluer la population des cervidés et de déterminer le plan de chasse annuel. Ce soir donc, chacun écoute en solitaire, sauf Yannick, le chef des chasseurs que j'accompagne. Nous nous postons dans un chemin creux, à l'abri du vent pour ne pas nous faire remarquer car, dit Yannick, « les cerfs ont une ouie et un odorat dix fois supérieurs aux nôtres ». Debout et immobiles au milieu du chemin, la vue bouchée de part et d'autre par de hauts talus, nous dressons l'oreille. Je n'entends rien. Ne vois rien. Ne sens rien. Et commence à trouver le temps long. Mais Yannick, à l'affût, ne relâche pas son attention. Il entend l'imperceptible, distingue le son d'un déplacement qu'il me signale du doigt, d'infimes bruissements de feuilles trahissant, pour lui seul, un jeune cerf non loin de nous. La zone est calme. Au bout d'une heure, mon guide décide de redescendre vers le parking. Là, près de la voiture, il retrouve la parole et ses cigarettes, et me raconte ce que je ne perçois pas : le relief des massifs, la manière dont le vent s'y faufile, porte les sons et influence le système de communication 
des cervidés. Si nous avons quitté notre poste, c'est aussi parce que Yannick sait que depuis le parking nous avons toutes les chances d'entendre l'écho de brames lointains. « Celui qu'on entend, là, il est au niveau du tracteur », commente-t-il, dirigeant mon écoute, « celui-là il est au bord de la route », « celui-là c'est celui qu'on a entendu tout à l'heure à 20h16, il descend vers Olivier », « il y en a encore un autre, là-dessous, près du chalet de la Frasse »... Si j'éprouve des difficultés à distinguer les cerfs, Yannick reconnaît chacun d'eux par les qualités sonores de leur brame et sait les situer instantanément. L'heure étant venue de rassembler les informations, nous gagnons la maison des chasseurs, une ancienne cabane de berger aménagée en lieu convivial, avec un poêle, une grande table, des chaises et le nécessaire pour festoyer entre pairs, réchauffer les liens après les battues, après avoir passé plusieurs heures seul à son poste à guetter l'apparition de la bête, plusieurs heures sans parler, baigné dans les sons de la forêt.

Dans la cabane, j'écoute les commentaires, observe la carte s'enrichir des numéros assignés à chacun des cerfs, et j'enregistre. Je ne suis pas là seulement pour mener une enquête ethnographique, je réalise aussi un documentaire sonore sur le brame. Une idée me traverse alors l'esprit : faire une sonographie de groupe, sur le modèle des photographies de groupe usuelles en pareilles circonstances, qui étayera le documentaire. J'imagine que, comme dans la vallée ariégeoise où je vis, les chasseurs imitent le brame du cerf. Contente de ma trouvaille, j'avance ma requête, l'échec est cuisant. Mines interloquées, silence gêné, quelle drôle de question, « heu... non... non... heu... ». Seul un chasseur se démarque du groupe, se vantant de savoir bramer avec un rouleau de papier essuie-tout en guise d'appeau, mais il n'y en a pas dans la cabane ; nous prenons rendez-vous pour que je l'enregistre au village. La soirée touchant à sa fin, chacun regagne sa voiture. Je remercie Yannick, qui s'attarde en bavardage le temps que chacun ait levé le camp. Manifestement il souhaite encore discuter. Quelque chose, semble-t-il, le préoccupe. Une fois seuls, il ne tarde pas à me mettre en garde contre ce chasseur « beau parleur » qui raconte souvent n'importe quoi pour faire l'intéressant. Or le porte-parole du groupe, le garant de ses valeurs et de sa cohésion, c'est Yannick: le « chef ». En me mettant ainsi en garde, il entend clairement me détourner de l'enregistrement prévu dans quelques jours au village. Pour mieux porter le coup, il précise encore que la pratique du frondeur n'est pas représentative, qu'elle serait en quelque sorte hors propos dans le documentaire - entendons par là tout ce que le montage sonore pourrait véhiculer comme représentations du monde de 
la chasse dans lequel Yannick a accepté de m'introduire ${ }^{7}$. À cette fin, il rétablit la vérité : « bien sûr que les chasseurs savent bramer, et nous n'avons pas besoin d'un rouleau de sopalin. Même Christine [la seule femme du groupe] commence à le faire $\gg$. Bramer à voix nue, lorsqu'on chasse, est un facteur d'intégration, une pratique qui marque un entre-soi. Pourquoi, alors, avoir refusé cette sonographie ? «On ne va pas bramer devant les autres » répond-il sans détour. Imiter le son du cerf en rut, appeler dans sa gorge et sa bouche le râle sauvage afin de le faire résonner à son tour dans la nature est certes une pratique partagée, mais qui se vit dans l'intimité et ne peut, en outre, être détachée d'un contexte qui donne sa puissance et son sens au son. Dans la cabane, devant le micro, à la demande d'une étrangère, l'idée même d'avoir à bramer de concert pour des auditeurs inconnus créé un malaise, soulignant à quel point le lien qui unit les chasseurs à ce son territorial et saisonnier ne peut être traité à la légère. Ma « gaffe », si l'on peut dire, embarrassante de prime abord, aura finalement eu pour conséquence heureuse d'attirer mon attention sur l'importance que les chasseurs accordent à la manière dont ils entrent en contact avec le son du brame. Le témoignage de Yannick qui, comme ses compagnons, brame lui aussi, invite donc à prolonger l'analyse du côté du processus d'attachement et des multiples opérations qui le constituent.

Après avoir dissipé les malentendus, le chasseur se met à raconter, pêle-mêle, ses aventures dans la forêt : lorsqu'il emmène ses enfants et, qu'après un pique-nique à la cabane, ils s'enfoncent dans les bois, la nuit, pour écouter et imiter le brame ; lorsqu'il va seul dans la forêt, hors période de brame et de chasse, pour observer, écouter bruire la nature et s'y immerger ; lorsque, un soir, un cerf s'est approché de lui si discrètement qu'il a sursauté quand l'animal s'est mis à bramer dans son oreille. Autant de situations diverses ressuscitées (re-suscitées) par l'évocation du brame et de son imitation, mais dont on peine parfois à percevoir le lien avec ce son spécifique. Sauf à le considérer comme l'empreinte sonore d'un processus, d'un « faire avec » et « par » les sons, une série d'opérations qui met savamment en place une manière particulière d'être dans et avec la forêt. Une acoustémologie commune aux chasseurs, qu'ils élaborent ensemble dans le creuset d'une pratique partagée et qu'ils nourrissent d'une expérience solitaire, d'un face à face ou plus

7. Cette précaution est probablement à mettre en lien avec le soin que prennent les chasseurs à donner de leur monde une image positive dans une société qui accepte de moins en moins leur conception de l'écologie. D'autant plus que le documentaire sonore « l'appel de la forêt » est produit par le Parc naturel régional du Haut-Jura, perçu par les chasseurs comme une institution dont les acteurs sont hostiles à leurs pratiques. 
exactement d'un bouche à oreille avec la bête - le cerf - figure emblématique de l'univers forestier.

Ce sont les opérations qui instaurent cette acoustémologie que Yannick me livre dans le désordre, après avoir essayé de m’y rendre sensible lors de notre écoute ; cette manière d'être et de connaître qu'il partage aussi avec ses enfants lorsqu'il les amène en forêt. Il ne s'agit pas seulement de passer un bon moment en famille, ni de découvrir un animal en particulier, mais aussi de transmettre une connaissance plus large de l'environnement ainsi qu'une manière singulière de s'y engager - la sienne et celle de ses amis chasseurs. Yannick prépare la relève en s'appuyant sur un son spécifique et son imitation. Dans la forêt, la nuit, alors qu'il fait noir et que les sonorités gutturales rendent palpables l'inquiétante étrangeté des lieux, il convie ses enfants à ce qui a tous les traits d'un jeu : il brame « pour faire venir le cerf », « pour le voir », et à leur tour les enfants s'essayent à bramer, imitant leur père qui imite le cerf. Ainsi, l'apprentissage sonore - le balbutiement des vocalisations rauques et puissantes caractéristiques du brame - est aussi l'une des premières étapes de l'apprentissage de la chasse. Il n'est peut-être pas inintéressant de constater la concordance entre cette étape et la dimension inaugurale de tous les brames humains : on ne brame pas n'importe quand mais quand les cerfs brament eux aussi. C'est en effet à la « saison des amours », qui est aussi, pour les chasseurs, celle de l'ouverture de la chasse, que l'on adopte l'expression sonore dominante en cette période. Or dans ce monde d'hommes, le brame ne sert-il pas aussi à penser la virilité, sans cesse éprouvée et rejouée durant la chasse ? Annonçant la chasse prochaine donc, le brame porte en lui les savoirs qui lui sont nécessaires. Leur acquisition a transformé la vie de Yannick. Il engage à présent ses enfants sur la même voie en les rendant attentifs non seulement au son, mais aussi, par son intermédiaire, à toute une série d'actions qui le rendent si important. C'est exactement ce qu'en Mongolie, Egi tente de faire percevoir à Laurent au retour d'une marche en montagne non loin d'Ulaanbaatar. Afin de poursuivre l'analyse de ces opérations qui font des sons des appuis, des soutiens et des jalons dans la connaissance intime des lieux, suivons l'ethnographe et son interlocuteur mongol sur les pentes de la montagne Bogd.

\section{Écouter}

En 2014, Egi réside à Ulaanbaatar, la capitale mongole où s'agroupe aujourd'hui un million et demi de personnes (soit la moitié de la population du pays) provenant des quatre coins de l'immense territoire national. Son pays natal, nutag, est à plus de mille kilomètres au nord-ouest de la grande ville. Le nutag est une notion fondamentale pour comprendre la sociabilité mongole. Elle regroupe à la fois l'idée d'un lieu qui a vu naître et grandir un individu - donc plutôt un 
chapelet de lieux liés par des itinéraires -, mais aussi le réseau de sociabilité auquel cet individu appartient (la parentèle bilatérale, les amis et autres accointances). À l'époque où prend place ce récit, Egi habite Ulaanbaatar depuis des années. Il y est investi dans diverses petites affaires plus ou moins légales et plus ou moins heureuses. Il a même été riche un jour grâce au commerce transfrontalier du transsibérien mais cela n'a duré qu’un jour. Tout lui a été volé le lendemain à son arrivée en gare d'Ulaanbaatar. Lorsque je me plains de la pollution sonore de la grande ville, Egi me rétorque que pour lui, entendre les klaxons rageurs des automobilistes équivaut à s'imaginer des centaines de personnes qui vaquent à leurs occupations et tentent de saisir une opportunité pour se faire une vie... Il s'en trouve stimulé et empli de cette énergie nécessaire à la traque aux bonnes affaires et à l'argent facile. Pour l'instant, précise-t-il, « il a saisi ses rêves par le tranchant », möröödliinhöö üzüürees atgasan. Et je sais qu'il emprunte cette puissante métaphore à une chanson à succès de l'époque. Le quotidien mongol est hautement perméable à la poésie. C'est là une de ses plus belles qualités. Ce jour-là, nous partons « enregistrer des sons », duu bichbeer garan, dans la montagne Bogd qui jouxte la ville au sud. Mais, fin mars, il n'y a rien à se mettre sous le micro. Il n'y a pas une once de vent lorsque nous gravissons les pentes - fait très rare en Mongolie, suffisamment pour qu'Egi le mentionne à plusieurs reprises en cours d'ascension - et à part le crissement de nos pas sur l'épaisse couche de neige glacée et ici ou là le graillement d'un choucas de Daourie ou d'un corbeau, la montagne est parfaitement muette... à notre grand dam !

Sur le chemin du retour, le vent se lève. Egi avise une série de tiges hautes enserrées dans une pellicule de givre et aux inflorescences séchées... Il lance : « si tu veux enregistrer quelque chose, enregistre ceci ! ». « Il y avait des plantes comme celle-là sur les pâturages de la Shishged (du nom de la plus large des rivières du pays darhad) ». Le discours d'Egi s'emballe. Il me raconte les pâturages de son enfance ; le vent qui se levait en mars ; la peur du blizzard ; la peur de ne pouvoir rentrer, la peur des loups... Il se souvient du nom de ces longues tiges : yargüi. Il se souvient du goût poivré de leur fleur dont il faisait profit pour agrémenter le pain et les fromages séchés qu'il avait emportés avec lui pour calmer la faim dans ces longues heures d'ennui sur les pâturages. Il se souvient qu'il passait au milieu des tiges en laissant glisser ses mains sur leurs extrémités. Il se souvient, et en se souvenant, il rejoue l'action pour accompagner (ou stimuler) sa réminiscence. Maintenant que je vois le geste, il me revient qu'Egi s'était livré au même jeu en montant. Je n'y avais pas vraiment prêté attention - je pense que lui non plus d'ailleurs. Le discours d'Egi égraine des toponymes accolés aux steppes, aux cols, aux sources et rivières. Autant de noms qui pointent vers les pâturages qui, pour reprendre son expression, l'« ont fait devenir homme », hün bolgoh. Dans toute 
la Mongolie, on envoie les enfants (filles et garçons) dès cinq ou six ans sur les pâturages, garder les chèvres et les moutons pour qu'ils deviennent autosuffisants et surmontent leur peur. Egi me raconte que ces yargüi poussaient en nombre, par taches éparses colorant de mauve la pâture. Il laisse sortir un son de sa bouche, un son qui mime les sonorités guindées des tiges saisies dans la glace et leurs frottements délicats quand elles s'entrecroisent dans le vent... « crrrrr ! ». Il me demande si je suis capable de l'entendre.

Je place les micros près des tiges et après quelques manipulations, je finis par capter un son malingre. Egi me demande de réécouter l'enregistrement au casque - j'espère secrètement que cela va relancer son discours - mais il enlève le casque et me le tend replié dans un silence dubitatif...

Entre cette brève description et celles des conduites de Gilbert White et de Yannick, une continuité forte se noue, en connexion directe avec un principe fondateur de l'acoustémologie : placer au centre de la focale l'attention portée vers les sons pour « se faire une place dans et à travers le monde ». Or, en suivant cette voie d'analyse, qu'entendons-nous ? Pas seulement un son (à son écoute, Egi me rend le casque un peu dépité). Il faut, c'est vrai, que le vent ou la main d'Egi fasse sonner les yargüi mais ce n'est pas suffisant. Alors que voyons-nous ? Pas seulement un homme non plus. Egi me raconte ce qui l'a amené à être ce qu'il est, mais en même temps son discours renvoie constamment à autre chose que lui. Ce qui transparaît plutôt de la description, c'est un homme qui fait ou mieux encore une séquence d'actions qui implique un homme (même plusieurs en réalité) et cet homme se dépense pour se rendre sensible aux suggestions que son trajet en montagne font naître. Il en saisit une qu'il sait être intimement liée à ce qu'il est devenu. Est-ce la réactivation d'une sensibilité sonore incorporée ? Si c'est seulement ça, décrire notre progression sur les pentes n'a presque aucune pertinence analytique (sauf celle de raconter une histoire ou de « rendre compte »ce qui est déjà énorme). Mais encore une fois, pourquoi alors me tend-il le casque sans avoir l'air d'éprouver quoi que ce soit ? C'est la description qui nous met sur la voie d'une réponse possible : parce que le son est bien sur la bande - tout constitué et prêt à être trituré à des fins d'analyse - mais qu'il ne laisse cette fois-ci aucune empreinte. Notre hypothèse est celle-ci : ce qui laisse une empreinte, ce qui impressionne, ce sont des cours d'actions car ils ont la faculté de modeler ceux qui s'y engagent (Hennion dans Small, 2019, p. 8). Ces cours d'actions (rappelez-vous la dépense d'énergie de White et toutes les procédures des chasseurs) laissent une empreinte d'autant mieux dessinée qu'ils convoquent les trois autres éléments de l'attachement : des relations sociales (de coprésence ou non), un objet qui répond et qui suggère de prendre en compte la singularité de sa réponse, et enfin, des altérations, même minimes, de ce que l'on est. 
Les cours d'actions dont nous parlons sont bien sûr sous influence culturelle. Citons trois éléments qu'Egi agence à sa manière. D'abord, il tourne son attention vers un son généré par le vent. Les formes $d u$ vent ${ }^{8}$ et ses résonnances avec différents matériaux constituent des points nodaux des paysages sonores mongols (des keynotes aurait dit Murray Schafer), auxquels on apprend à se rendre attentif dès le plus jeune âge en Mongolie. Ensuite, il est d'usage depuis des siècles - au moins depuis l'époque de la Mongolie sous juridiction sino-mandchoue (1691-1911) que les jeunes garçons gagnent la montagne mongole pour être transformés. Ce comportement, Egi l'évoque parfois en me parlant des sain er (les « hommes bons »), ces robins des bois mongols qui se réfugiaient en montagne et menaient une guerre d'usure aux troupes de l'« envahisseur » mandchou. Enfin, l'impact du son des yargüi sur Egi est lié à la marque laissée par un dispositif éducatif mongol - la garde du petit troupeau par les jeunes enfants. Dans les zones rurales, aucun « morveux », nustai, n’y échappe. Il y a donc là une sorte de « gabarit culturel $^{9} \gg$ (Crawford, 2016, p. 55) qui encadre les efforts déployés par Egi pour saisir les suggestions sonores que la montagne lui tend.

Je pense avoir compris des années plus tard, à la suite d'échanges sur Skype, ce qu'Egi faisait en cheminant sur la montagne Bogd. Il ne fuyait pas le tintamarre de la ville. Pour lui ce maelström est une vraie stimulation. Il ne partait pas non plus contempler la nature (cela n'a pris du sens que très récemment en Mongolie). Lorsqu'il était saisi de nostalgie pour son pays natal, plutôt que de subir ces « pensées qui assèchent »-comme les Mongols disent -, il gravissait les pentes et allait au contact de suggestions perceptuelles (en l'occurrence sonores) pour

8. L'expression est empruntée au titre du recueil de textes de lettrés chinois ( $\mathrm{du}^{\mathrm{e}}$ au $\mathrm{XVIII}^{\mathrm{e}}$ siècle) sur les paysages traduits par Martine Vallette-Hémery. Parmi eux le texte intitulé « Le pavillon du vent dans les Pins » (Vallette-Hémery, 2007, p 73-79) du poète et militaire Liu Ji (1311-1375) est étonnement proche de ce que je pense être la sensibilité générale des Mongols aux sonorités produites par le vent. Sa lecture m’a été conseillée par Françoise Lauwaert après m'avoir entendu parler des tribulations d'Egi sur la montagne. Je la remercie vivement de m'avoir signalé ce texte magnifique.

9. Adopter une ligne de conduite ou échafauder une manière de faire sont choses d'autant plus aisées qu'elles bénéficient des contraintes de gabarits culturels, c'est-à-dire d'habitudes de penser et de percevoir saillantes et partagées dans une population donnée. «Bénéficier de contraintes »; ce quasi oxymore montre bien que ce qui se trouve au cœur de la notion de gabarit culturel est l'habileté à agencer des éléments culturels et à s'en servir comme de ressources ou d'appuis pour faire « au mieux » au regard de la situation dans laquelle un individu se trouve plongé. 
que celles-ci le marquent à nouveau ${ }^{10}$. Je m'avance sans doute beaucoup, mais je pense que l'empreinte du son des yargüi lui permettait d'orienter ses pensées et de les faire porter sur le chemin de vie déjà parcouru et sur celui à venir.

Egi est loin maintenant. Il vit à la frontière chinoise, dans le Gobi. À ma question, pianotée sur le clavier de mon ordinateur, de savoir s'il trouve là des yargüi qu'il peut aller écouter, il répondra laconiquement : «C'est sec ici. Il n’y a pas de yargüi. Mon nutag me manque encore plus qu'avant. »

\section{Réentendre : l'écho de l'écoute et l'empreinte sonore}

$\ll[\ldots]$ souvenez-vous que dans le champ de l'observation, le hasard ne favorise que les esprits préparés. »

Louis Pasteur, 1854

Que pouvons-nous retirer de ces moments passés avec des personnes qui soignent une certaine forme d'écologie de l'attention ? Est-il possible de faire entrer en résonance des données si hétéroclites ? Pour conclure, nous proposons un modèle dont la pertinence reste largement à éprouver mais qui présente certains avantages. Celui, par exemple, de mettre en avant tout ce qu'il faut faire pour connaître plus intimement et être au monde avec ou par les sons. Ce modèle fait par conséquent la part belle à la description de séquences d'action et prend en compte ce qui se passe en amont de la rencontre entre des gens et des sons sans évider la richesse de ce contact, ni ses effets en aval.

Les naturalistes ont une technique ingénieuse qu'ils appellent le « revoir » (Aubrun, 2019). Dans le cadre de l'étude des comportements des mammifères en danger, elle consiste à ameublir la terre là où on suppose que l'animal - ce « corps d'habitudes » (Morizot, 2018, p. 90) - passera à nouveau. Le but est d'obtenir une empreinte parfaitement dessinée qui livrera le maximum d'informations sur

10. Remarquons encore une chose même si nous n'en ferons rien ici : le flux de cette succession d'actions est séquencé d'une manière très similaire à ce que John Dewey conçoit comme un fondement de l'esthétique dans sa tentative de refonte de la notion. Une expérience esthétique est caractérisée - entre autre chose mais crucialement - par la transitivité entre des phases agies et des phases subies et par la conscientisation des articulations qui mènent de l'une de ces phases à l'autre (Dewey, 1934, p. 35-57). Egi grimpe dans la montagne pour ne pas être sous l'emprise de pensées néfastes, il se voit soumis aux aléas des suggestions perceptuelles qui vont se présenter, il réagit à l'une d'elles et s'y appuie pour se penser autrement et enrichir sa relation à un environnement qu'il singularise, etc. 
les caractéristiques et le comportement de l'espèce en général et d'un individu en particulier. Ce qui est intéressant pour notre propos dans le « revoir », c'est que l'empreinte arrive au terme d'un processus qui nécessite toute une mise en place : savoir où et quand aller la chercher (soit des connaissances préalables et plus ou moins actualisées), vouloir aller la chercher (soit une intention logée dans le cadre d'une pratique qui donne du sens à ce que l'on fait), préparer le terrain (soit se déplacer vers le lieu où l'animal a déjà laissé des traces, trouver l'endroit le plus propice, sortir son râteau et ameublir le sol). Enfin, il faut aussi un peu de chance pour que tout cela prenne corps, c'est-à-dire pour que l'empreinte soit à la fois dans la terre mais que l'on en retrouve aussi la trace dans l'enthousiasme des naturalistes rassemblés autour d'elle. L'affaire n'est jamais entendue à l'avance. L'animal viendra, ou pas, passera sur la terre meuble ou juste à côté, piétinera à ce point le « revoir » que plus rien ne sera visible et lisible ou, au contraire, aura l'élégance de ne laisser qu'une empreinte de chacun de ses membres. Si tel est le cas, le résultat donnera raison au naturaliste d'avoir choisi cet endroit dans la mise en place de son « revoir » et ouvrira la possibilité d'une amélioration des connaissances éthologiques. La forme de l'empreinte, sa profondeur et sa disposition diront probablement plus au naturaliste que ce qu'il ne sait déjà, à son immense satisfaction.

$\mathrm{Au}$ terme du parcours proposé, l'empreinte sonore nous apparaît être en quelque sorte la technique du « réentendre » ou l'écho de l'écoute. Sur ce point nos vignettes se répondent : réentendre le brame pour Yannick, ses enfants et ses chasseurs, réentendre le vent dans les yargüi pour Egi. Dans les deux cas, ce « réentendre » demande toute une mise en place qui fait entièrement partie de la « puissance d'affecter » (Despret, 2019, p. 64) du son. Nos interlocuteurs se trouvent bien dubitatifs en face d'une séquence sonore détachée des opérations qui lui donnent cette force et disjointe de leur manière de participer à son avènement. En bref, ils n'éprouvent plus grand-chose lorsque nous leur proposons une autre mise en place, à mille lieues celle-ci, de leur façon d'habiter le monde en s'y frayant un chemin par (et en partie pour) les sons. Comme dans la technique $\mathrm{du}$ 《 revoir », l'empreinte sonore nous apparaît comme la résultante finale d'une série d'opérations qui lui permettent de s'imprimer. Elle est bien la trace laissée par un contact entre des sons et des gens. Mais ces contacts sont rarement le fruit de la rencontre heureuse entre une sensibilité incorporée et des sons qui l'activent. Ils sont ce qui arrive lorsque des gens adoptent des lignes de conduites canalisées et potentialisées par des gabarits culturels, auxquelles la vie sociale et des intérêts communs donnent une orientation et que des personnes apprennent à s'approprier soit collectivement soit de manière plus solitaire. Faire avec et faire pour les sons ; être avec et être par les sons. Cette ligne de conduite n'est jamais 
donnée d'avance. Dans cet article, nous avons mis l'accent sur les menus réglages quotidiens qu'une telle attitude nécessite. Et pour ce faire, nous avons simplement suivi la piste d'empreintes sonores laissées par des séquences d'actions au cours desquelles, dans la vallée de Selborne, dans les forêts du mont Bosavi, sur les pentes boisées d'un massif jurassien ou celles « rocailleuses » d'une montagne de Mongolie se sont avancés, jusqu'à entrer en contact, des sons et des personnes...

\section{Bibliographie}

Aubrun Brian, 2019, Le Suivi de l'ours dans les Pyrénées. Utilisation et production du savoir, Mémoire de master non publié, Université Toulouse Jean Jaurès.

Certeau Michel (de), 1990, L'Invention du quotidien. Tome 1. Arts de faire, Gallimard (coll. Folio essais), Paris, 416 p.

Citton Yves, 2014, Pour une écologie de l'attention, Seuil (coll. La couleur des idées), Paris, $320 \mathrm{p}$.

Crawford Matthew, 2016, Contact. Pourquoi nous avons perdu le monde et comment le retrouver, La Découverte, Paris, 352 p.

Despret Vinciane, 2019, Habiter en oiseau, Actes Sud, Arles, 224 p.

Dewey John, 1934, Art as Experience, Minton, Balch \& Company, New York, $355 \mathrm{p}$.

Feld Steven, 2003, "A Rainforest Acoustemology" in Bull M. \& BACK L. (eds.), The Auditory Culture Reader, Berg, Oxford/New York, pp. 223-240.

Hennion Antoine, 2003, « Ce que ne disent pas les chiffres. Vers une pragmatique du goût » in Donnat O. (dir.), Les Publics, politiques publiques et équipements culturels, Éditions de la FNSP, Paris, p. 287-304.

Hennion Antoine, 2007, "Those Things That Hold Us Together: Taste and Sociology” in Cultural Sociology, n ${ }^{\circ}$ 1(1), pp. 97-114.

Ingold Tim, 2018, Faire. Anthropologie, archéologie, art et architecture, Éditions Dehors, Bellevaux, 320 p. 
Acoustémologie et empreinte sonore. Faire aVec et faire par les sons.

ÊTRE AVEC ET ÊTRE PAR LES SONS

Marie BALTAZAR \& Laurent Legrain

Laurens Camille, 2011, Philippe, Stock, Paris, 320 p.

Latour Bruno, 2012, Enquête sur les modes d'existence. Une anthropologie des Modernes, La Découverte, Paris, 504 p.

Morizot Baptiste, 2018, Sur la piste animale, Actes Sud, Arles, 208 p.

Schafer R. Murray, 1977, The Soundscape. The Tuning of the World, Destiny books, Vermont/ Rochester, 320 p.

Small Christopher, 2019, Musiquer. Le sens de l'expérience musicale, Philarmonie de Paris éditions, Paris, 448 p.

Tournier Michel, 2004, Journal extime, Gallimard (coll. Folio), Paris, 272 p.

Vallette-Hémery Martine, 2007, Les Formes du vent. Paysages chinois en prose, Albin Michel, Paris, 188 p.

White Gilbert, 2011, Histoire naturelle de Selborne, Le mot et le reste, Marseille, $320 \mathrm{p}$.

Résumé : Au XVIII ${ }^{\mathrm{e}}$ siècle, un pasteur anglais déclame des vers de Virgile dans une vallée de sa paroisse et s'émerveille de la puissance de l'écho. En 2018, des chasseurs se réunissent sur le massif du Massacre dans le Jura pour se répartir des points d'écoute avant de s'en aller, seuls dans la nuit qui tombe, se baigner dans les sonorités du brame des cerfs. En 2014, un jeune homme gravit la montagne qui jouxte les quartiers les plus au sud d'Ulaanbaatar et se rend attentif à la sonorité délicate du frottement de tiges engoncées dans le givre printanier. Ces vignettes ethnographiques, très disparates, se font pourtant écho et cet article met l'accent sur cette correspondance : leur capacité à porter sur le devant de la scène descriptive une somme de petites opérations que subsume le verbe « écouter ». Dans le sillage de l'épistémologie acoustique et de l'attachement, les auteurs proposent de modéliser la notion d'《empreinte sonore » non pas en pensant l'empreinte comme la simple réactivation d'une sensibilité incorporée au préalable - bien qu'il soit difficile de nier totalement l'existence d'un tel processus - mais en ethnographiant tout le travail consenti pour « faire avec » et « être par » les sons.

Mots-clefs : anthropologie, attachement, épistémologie acoustique, son, attention, chasse, Mongolie, habiter. 
Abstract: In the $18^{\text {th }}$ Century, an English pastor recites verses of Virgil in a valley in his parish and marvels at the power of the echo. In 2018, hunters come together on the Massacre Massif in the Jura to divide up listening points before going off, alone as night fell, to bathe in the sound of the cries of deer. In 2014, a young man climbs the mountain that adjoins the southernmost neighborhoods of Ulaanbaatar and makes bimself attentive to the delicate sonority of the rubbing of stems bundled up by the springtime frost. These ethnographic vignettes, though quite different, nonetheless echo one another. This article highlights their correspondence, namely their shared capacity to bring to the forefront of the descriptive stage a sum of the small operations that the verb "listen" subsums. In the wake of acoustic epistemology and the notion of attachment, the authors propose to model the notion of "sonic imprints", not by considering the imprint as a simple reactivation of an already incorporated sensibility-though it would be difficult to deny its existence-but rather by ethnographizing all the work needed to "do with" and "be by" sounds.

Keywords: anthropology, attachment, acoustemology, sound, attention, hunting, Mongolia, dwelling.

\section{Notes sur les auteurs}

Marie Baltazar a travaillé sur les processus de construction des identités musiciennes, notamment dans le milieu de l'orgue. Les notions d'apprentissage et de construction des savoirs sont au centre de ses recherches.

Laurent Legrain a mené ses recherches en Mongolie et parmi les groupes d'amateurs de jazz en Belgique. Les thèmes qu'il y aborde gravitent autour de la transmission d'un lien fort aux sonorités de la musique, du langage et de l'environnement. 